\title{
A New Aspect for Some Sequence Spaces Derived Using the Domain of the Matrix $\widehat{\widehat{B}}$
}

\author{
Murat Candan \\ Department of Mathematics, Faculty of Science, İn̈nü University, Battalgazi-Malatya, Turkey
}

\author{
Article Info \\ Keywords: Matrix domain of a se- \\ quence space, Schauder basis, $\beta$ - and \\ $\gamma-$ duals and matrix transformations \\ 2010 AMS: 46A45, 40C05 \\ Received: 2 October 2021 \\ Accepted: 11 February 2022 \\ Available online: 23 February 2022
}

\begin{abstract}
This study serves for analysing algebraic and topological characteristics of the sequence spaces $X(\widehat{\widehat{B}}(r, s))$ constituted by using non-zero real number $r$ and $s$, where $X$ denotes arbitrary of the classical sequence spaces $\ell_{\infty}, c, c_{0}$ and $\ell_{p}(1<p<\infty)$ of bounded, convergent, null and absolutely $p$-summable sequences, respectively and $X(\widehat{\widehat{B}})$ also is the domain of the matrix $\widehat{\widehat{B}}(r, s)$ in the sequence space $X$. Briefly, the $\beta$ - and $\gamma$-duals of the space $X(\widehat{\widehat{B}})$ are computed, and Schauder bases for the spaces $c(\widehat{\widehat{B}}), c_{0}(\widehat{\widehat{B}})$ and $\ell_{p}(\widehat{\widehat{B}})$ are determined, and some algebraic and topological properties of the spaces $c_{0}(\widehat{\widehat{B}}), \ell_{1}(\widehat{\widehat{B}})$ and $\ell_{p}(\widehat{\widehat{B}})$ are studied. Additionally, it is observed that all these spaces have some remarkable features, including the classes $\left(X_{1}(\widehat{\widehat{B}}): X_{2}\right)$ and $\left(X_{1}(\widehat{\widehat{B}}): X_{2}(\widehat{\widehat{B}})\right)$ of infinite matrices which are characterized, in which $X_{1} \in\left\{\ell_{\infty}, c, c_{0}, \ell_{p}, \ell_{1}\right\}$ and $X_{2} \in\left\{\ell_{\infty}, c, c_{0}, \ell_{1}\right\}$.
\end{abstract}

\section{Background and historical developments}

One of the non-classical approaches when building new sequence space used recently in summability is that of working with any infinite matrix. Although this technique is not easy, it provides a quick technique in obtaining certain results if the inverse of an infinite matrix is present. In addition to the different aspects of this technique used in the listed references at the end of the article, much more detailed information can be found in the five books of Başar [1], Başar and Dutta [2], Mursaleen and Başar [3], Mursaleen [4] and Malafosse et al. [5] published recently. We now remind some basic definitions and conclusions, which we will mainly use in the following sections. Any $x$ sequence in $X$ is a transformation $x: \mathbb{N} \rightarrow X$, where $X$ is a non-empty set. The collection of all real or complex number sequences forms a vector space which we denote by $w$, under the operations of coordinate-wise addition and well-known scalar multiplication. The subspaces of $w$ are important in such applications because each of them is called a sequence space. We denote $\ell_{\infty}, c, c_{0}$ and $\ell_{p}$ for the classical sequence spaces of all bounded, convergent, null and absolutely $p$-summable sequences, respectively. $b v$ is the space consisting of all sequences $\left(x_{k}\right)$ such that $\left(x_{k}-x_{k+1}\right)$ in $\ell_{1}$ and $b v_{0}$ is the intersection of the spaces $b v$ and $c_{0}$ where $k \in \mathbb{N}$. Unless otherwise stated, all other chapters shall also be applicable to $p, q>1$ with $p^{-1}+q^{-1}=1$ and utilize the fact that each term having negative subscript equals to zero.

Let us remember the definition of another concept we need. Given an infinite matrix $A=\left(a_{n k}\right)$ of complex numbers $a_{n k}$, where $n, k \in \mathbb{N}$, for any sequence $x$, we write

$$
(A x)_{n}:=\sum a_{n k} x_{k} ; \quad\left(n \in \mathbb{N}, x \in D_{00}(A)\right),
$$

where $D_{00}(A)$ denotes the subspace of $\omega$ consisting of $x \in \omega$ for which the sum exists as a finite sum. For simplicity in illustration, here and wherever after that, the summation without limits runs from 0 to $\infty$. 
Now, let us continue by giving the definition of a matrix transformation between arbitrary sequence spaces $X, Y$ that will be required in the following sections. Having supposed that $Y$ is a normed sequence space, it is natural to consider the question of whether or not the sum in (1.1) is converges in the norm $Y$ for $x \in X$, for this situation we write $D_{Y}(A)$. In this meaning, $(X: Y):=\left\{A: X \subseteq D_{Y}(A)\right\}$ is written for the space of all matrices that satisfy the condition mentioned in the previous row, which send the all of $X$ into $Y$.

We need a definition and some of its results, which provide some advantage in our paper. A matrix $T=\left(t_{n k}\right)$ is said to be a triangle if $t_{n k}=0$ for $k>n$ and $t_{n n} \neq 0$ for all $n \in \mathbb{N}$. As the immediate consequences of this concept, we have the following useful results. Let $U$ and $V$ two triangle matrix and $x$ any sequence then $U(V x)=(U V) x$ is valid. Moreover, the inverse of such a matrix is always uniquely exist and at the same time has a triangle matrix as well. In a practical way, we can obtain, if the inverse of matrix $U$ is $V$, then $x=U(V x)=V(U x)$ is always valid for all $x \in \omega$.

Now, we shall be concerned with certain properties of difference sequences. First of all, we define and discuss briefly the meaning of the concept of difference sequence spaces. In 1981 Krzmaz [6] defined new sequence spaces using the sequence $\left(x_{k}-x_{k+1}\right)$ instead of working directly by a sequence $x=\left(x_{k}\right)$. Let $X$ denote an arbitrary well-known classical sequence spaces $\ell_{\infty}, c$ or $c_{0}$. Kizmaz [6] defined the sequence spaces $X(\Delta)=\left\{x=\left(x_{k}\right) \in w: \Delta x \in X\right\}$ where $\Delta x=\left(x_{k}-x_{k+1}\right)$ and also showed that these are the Banach spaces with the norm $\|x\|_{\Delta}=\left|x_{1}\right|+\|\Delta x\|_{\infty} ; \quad x=\left(x_{k}\right) \in X$. These spaces are called difference sequence spaces. His new method is an expansion of the classical sequence spaces, which are probably more familiar to most readers. In other words, the inclusion relation $X \subset X(\Delta)$ is strictly valid. Shows that many facts about difference sequence spaces. K1zmaz [6] obtained almost basic algebraic and topological properties in his work, including the $\alpha-, \beta-$ and $\gamma$-duals of the difference sequence spaces and $(X(\Delta): Y)$ and $(Y: X(\Delta))$ of infinite matrices, where $X, Y \in\left\{\ell_{\infty}, c\right\}$. Following Kizmaz's Technique, Et [7] defined the sequence spaces $X\left(\Delta^{2}\right)=\left\{x=\left(x_{k}\right) \in w: \Delta^{2} x \in X\right\}$ where $\Delta^{2} x=\left(\Delta^{2} x_{k}\right)=\left(\Delta x_{k}-\Delta x_{k+1}\right)$ and $X \in\left\{\ell_{\infty}, c, c_{0}\right\}$. In 1987 Sarıöl [8] introduced a new difference sequence spaces $X\left(\Delta_{t}\right):=\left\{x=\left(x_{k}\right) \in w:\left(\Delta_{t} x\right)=\left[k^{t}\left(x_{k}-x_{k+1}\right)\right] \in X\right.$ for $\left.t<1\right\}$ which more complicated than the spaces of K1zmaz [6] and he observed its some algebraic and topological properties, where $X \in\left\{\ell_{\infty}, c, c_{0}\right\}$. It is the fact that it is easy to get both of the following inclusion relationships are valid: $X\left(\Delta_{t}\right) \subset X(\Delta)$, if $0<t<1$ and $X(\Delta) \subset X\left(\Delta_{t}\right)$, if $t<0$. Simultaneously, the sequence spaces $X(p, \Delta)$ which are expanded from the previous ones defined by Ahmad and Mursaleen [9] and they studied various problems. Almost two years later, Malkowsky [10] introduced the sequence spaces $\ell_{\infty}(p, \Delta), c_{0}(p, \Delta)$ and specified the Köthe-Toeplitz duals of them and proved characterization of the matrix transformations discussed in [9]. Later on, Choudhary and Mishra [11] examined certain characteristic of the sequence space $c_{0}\left(\Delta_{t}\right)$, for $t \geq 1$. In the same year, a characterization of $B K$-spaces involving a subspace which is isomorphic to $s c_{0}(\Delta)$ with respect to matrix maps obtained by Mishra [12] and a sufficient situation of a map from $s \ell_{\infty}(\Delta)$ into a $B K$-space for being compact operator. He proved that arbitrary matrix from $s \ell_{\infty}(\Delta)$ is compact, where $s X(\Delta)=\left\{x=\left(x_{k}\right) \in w:\left(\Delta x_{k}\right) \in X, x_{1}=0\right.$ for $X=\ell_{\infty}$ or $\left.c_{0}\right\}$. In the year 1996, Mursaleen et al. [13] interested in introducing and examining the sequence space $\ell_{\infty}\left(p, \Delta_{r}\right)=\left\{x=\left(x_{k}\right) \in w: \Delta_{r} x \in \ell_{\infty}(p)\right\}, \quad(r>0)$. Gnanaseelan and Srivastava [14] introduced and investigated the spaces $X(z, \Delta)$ for a non-complex numbers $z=\left(z_{k}\right)$ satisfying the following three conditions

(i) $\frac{\left|z_{k}\right|}{\left|z_{k+1}\right|}=1+O(1 / k)$ for each $k \in \mathbb{N}_{1}=\{1,2,3, \ldots\}$.

(ii) $k^{-1}\left|z_{k}\right| \sum_{i=0}^{k}\left|z_{i}\right|^{-1}=O(1)$.

(iii) $\left(k\left|z_{k}^{-1}\right|\right)$ is a sequence of positive numbers increasing monotonically to infinity.

Malkowsky [15] described the spaces $X(z, \Delta)$ for any fixed sequence $z=\left(z_{k}\right)$ not having restriction upon $z$ in the same year. The author has also gave the proof of the fact that the sequence spaces $X(u, \Delta)$ are $B K$ - spaces having the norm given by $\|x\|=\sup _{k \in \mathbb{N}}\left|u_{k-1}\left(x_{k-1}-x_{k}\right)\right|$ with $u_{0}=x_{0}=1$. Subsequently, Gaur and Mursaleen [16] defined a more general space $S_{r}(p, \Delta)$ using the space $S_{r}(\Delta)$, where $S_{r}(\Delta)=\left\{x=\left(x_{k}\right) \in w:\left(k^{r}\left|\Delta x_{k}\right|\right) \in c_{0}(p)\right\}, \quad(r \geq 1)$ and they characterized both the matrix classes $\left(S_{r}(p, \Delta): \ell_{\infty}\right)$ and $\left(S_{r}(p, \Delta): \ell_{1}\right)$. Almost simultaneously and independently of each other; Malkowsky et al. [17], and Asma and Çolak [18] defined the sequence spaces $X(p, u, \Delta)$ which is a generalization of the sequence spaces $X(u, \Delta)$ and examined some of their properties for $X \in\left\{\ell_{\infty}, c, c_{0}\right\}$. In 2001, the matrix classes $(\Delta X: Y)$ and $(\Delta X: \Delta Y)$ are characterized by Malkowsky and Mursaleen [19], where $X \in\left\{c_{0}(p), c(p), \ell_{\infty}(p)\right\}$ and $Y \in\left\{c_{0}(p), c(p), \ell_{\infty}(p)\right\}$.

What is very important for us and the framework of this study is the matrix domain. For this reason, in this paragraph is presented the definition of it. The connection between any sequence space $X$ and any limitation matrix $A$ as below lets us the concept known as a matrix domain $X_{A}$ to describe;

$$
X_{A}:=\left\{x=\left(x_{k}\right) \in \omega: A x \in X\right\}
$$

which gives a sequence space. If $X$ is a sequence space, then the continuous dual $X_{A}^{*}$ of the space $X_{A}$ is defined by $X_{A}^{*}:=\{f$ : $\left.f=g \circ A, g \in X^{*}\right\}$

Now, the matrix domain concept is briefly analyzed. There may be a relationship between the new sequence space $X_{A}$ made up of using the limitable matrix $A$ and the original sequence spaces $X$. This relationship can come across us in different ways, depending on the choice of $X$ and $A$. Let us explain what we have said with examples. In fact, we find the relation if $X \in\left\{\ell_{\infty}, c, c_{0}\right\}$ then we obtain that the inclusion we seek $X_{S} \subset X$ is strictly valid where $S=\left(s_{n k}\right)$ is the summation matrix described by $s_{n k}=\left\{\begin{array}{lll}1 & , & (0 \leq k \leq n), \\ 0 & , & (k>n) .\end{array}\right.$

But, if $X$ is an element of the set $X \in\left\{\ell_{\infty}, c, c_{0}, \ell_{p}\right\}$ then one can easily see that 
the inclusion $X \subset X_{\Delta^{(1)}}$ is strictly valid, where $\Delta_{n k}^{(1)}=\left\{\begin{array}{cl}(-1)^{n-k}, & (n-1 \leq k \leq n), \\ 0 \quad, \quad 0 \leq k<n-1 \text { or }(k>n) .\end{array}\right.$ However, when we describe $X:=c_{0} \oplus$ spant together with $t=\left((-1)^{k}\right)$ namely; $x \in X$ only when $x:=z+\lambda x$ for some $z \in c_{0}$ and some $\lambda \in \mathbb{C}$, and take into consideration the given matrix $A$ together with the rows $A_{n}$ given by $A_{n}:=(-1)^{n} e^{(n)}$ for all $n \in \mathbb{N}$, then we are going to obtain $A e=t \in X$ but $A t=e \notin X$, resulting in the conclusion that $t \in X \backslash X_{A}$ and $e \in X_{A} \backslash X$ in which $e=(1,1,1, \ldots)$ and $e^{(n)}$ is a given sequence of which its unique term different from zero is a 1 found in the $n$th position for every $n \in \mathbb{N}$. In view of this explanation, both of the sequence spaces $X_{A}$ and $X$ overlap each other; however, neither of them contains the other. The tendency to built a new sequence spaces from the old ones is a widely used method. One of the most popular of these methods is obtain new sequence spaces using the matrix domain of a certain limitation method.

Today, the tendency for building a new sequence spaces via matrix domain and its extensions in summability theory is really reaching a wide range. It is recommended to look at the references to see what a profusion of problems it solves and what a wide range of fields now it uses in the different mathematical models that bring understanding about it.

In analogy with the difference sequence spaces that you have seen in the paper of Kizmaz [6], the sequence spaces defined by Kirişçi and Başar [20] are a generalization of the previously defined spaces. With the $r$ and $s$ being non-zero real numbers, Kirişçi and Başar [20] constructed the respective spaces using the matrix $\widehat{B}$ defined as below

$$
\widehat{b}_{n k}(r, s)= \begin{cases}r \quad, \quad(k=n), \\ s \quad, \quad(k=n-1), \\ 0 \quad, \quad(0 \leq k<n-1 \text { or } k>n),\end{cases}
$$

for all $k, n \in \mathbb{N}$. We would like to multiply the matrix $\widehat{B}$ by itself and we want to use it. This old approach is nothing new. Here is the formal statement. Let $r, s$ be non-zero reel numbers and define the band matrix $\widehat{\widehat{B}}(r, s)=\left\{\widehat{\widehat{b}}_{n k}(r, s)\right\}$ by

$$
\widehat{b}_{n k}(r, s)=\left\{\begin{array}{cll}
r^{2} & , & (n=k), \\
2 r s & , & (k=n-1), \\
s^{2} & , & (k=n-2), \\
0 & , & \text { otherwise, }
\end{array}\right.
$$

for all $k, n \in \mathbb{N}$. To simplify the notation let us write $\widehat{\widehat{B}}(r, s)=\widehat{\widehat{B}}$ and so forth. We must state the fact that here, the newly defined matrix $\widehat{\widehat{B}}$ can be derived from the triple band matrix $B(r, s, t)$ used by Sönmez [21] and the main results are obtained independently from the paper of Sönmez [21].

Again in analogy with the sequence spaces that one can see in the paper of Kirişçi and Başar [20], the sequence spaces introduced by Candan [22] are a generalization of the previously defined spaces. With $\widetilde{r}=\left(r_{n}\right)_{n=0}^{\infty}$ and $\widetilde{s}=\left(s_{n}\right)_{n=0}^{\infty}$ being convergent sequences of positive real numbers. Candan [22] introduced the respective spaces using the matrix $\widetilde{B}=\widetilde{B}(\widetilde{r}, \widetilde{s})=\left\{b_{n k}(\widetilde{r}, \widetilde{s})\right\}$ defined as below

$$
b_{n k}(\widetilde{r}, \widetilde{s})=\left\{\begin{array}{cll}
r_{n} & , \quad(k=n), \\
s_{n} & , \quad(k=n-1), \\
0 \quad, & (0 \leq k<n-1 \text { or } k>n),
\end{array}\right.
$$

for all $k, n \in \mathbb{N}$. The $\widetilde{B}(\widetilde{r}, \widetilde{s})-$ transforms of a sequence $x=\left(x_{k}\right)$ is $\widetilde{B}(\widetilde{r}, \widetilde{s})(x)=r_{k} x_{k}+s_{k-1} x_{k-1}$ for all $k \in \mathbb{N}$. In the last decade, Candan [22]-[24] has worked on many different studies using the $\widetilde{B}$ matrix.

The main emphasis in this paper is going to be on defining the sequence space $X(\widehat{\widehat{B}})$ and continuing with explanations of the properties accounting for their importance in scientific work, and determining the $\beta-$ and $\gamma$-duals of the spaces, in which $\widehat{\widehat{B}}$ denotes the any of the classical spaces $\ell_{\infty}, c, c_{0}$ or $\ell_{p}$ and $\widehat{\vec{B}}$ is the band matrix $\widehat{\vec{B}}(r, s)$. Moreover, the Schauder basis for the space $c(\widehat{\widehat{B}}), c_{0}(\widehat{\widehat{B}})$ and $\ell_{p}(\widehat{\widehat{B}})$ are obtained, and some topological properties of the spaces $c(\widehat{\widehat{B}}), c_{0}(\widehat{\widehat{B}})$ and $\ell_{p}(\widehat{\widehat{B}})$ are studied Finally, some classes of matrix mappings on the space $X(\widehat{B})$ are calculated.

The present paper is roughly composed as follows: In Section 1, we explain the kinds of sequence spaces that arise in scientific study, including basic concepts, historical developments of some subjects and matrix domain etc. In Section 2, the domain $X(\widehat{\widehat{B}})$ within the sequence space $X$ with $X \in\left\{\ell_{\infty}, c, c_{0}, \ell_{p}\right\}$ is going to be introduced, and the $\beta-$ and $\gamma-$ duals of $X(\widehat{\widehat{B}})$ will be determined. The Schauder basis of the spaces $c(\widehat{\widehat{B}}), c_{0}(\widehat{\widehat{B}})$ and $\ell_{p}(\widehat{\widehat{B}})$ are given after a proof is given about under which conditions the equality $X=X(\widehat{\widehat{B}})$ and inclusion $X \subset X(\widehat{\widehat{B}})$ are valid. In final section, some topological properties of those spaces $c_{0}(\widehat{\widehat{B}}), \ell_{1}(\widehat{\widehat{B}})$ and $\ell_{p}(\widehat{\widehat{B}})$ having $p>1$ are investigated. In Section 3 , a general theorem which characterizes the matrix transformations from the domain of a triangle matrix into any sequence spaces is stated and also proven. To present the application of this fundamental theorem, a table is given showing the necessary and sufficient conditions for a matrix transformations from $X(\widehat{\widehat{B}})$ to $Y$ in which $X \in\left\{\ell_{\infty}, c, c_{0}, \ell_{p}\right\}$ and $Y \in\left\{\ell_{\infty}, c, c_{0}, \ell_{p}\right\}$. 


\section{Some new sequence spaces derived by the domain of the matrix $\widehat{\widehat{B}}(r, s)$}

Section 2 is devoted to a quick review of the newly defined sequence spaces derived by using a band matrix $\widehat{\widehat{B}}$ defined above and its various properties. Briefly, the subject of this section is the definition the sequence spaces $\ell_{\infty}(\widehat{\widehat{B}}), c(\widehat{\widehat{B}}), c_{0}(\widehat{\widehat{B}})$ and $\ell_{p}(\widehat{\widehat{B}})$, and it is seen that they are norm isomorphic to the spaces well-known classical sequence spaces. Moreover, one of the typical applications includes calculating the $\beta$ - and $\gamma$-duals of the spaces. Armed with elementary facts given earlier in the article, we can now describe the spaces as follows;

$$
\begin{gathered}
\ell_{\infty}(\widehat{\widehat{B}}):=\left\{x=\left(x_{k}\right) \in w: \sup _{k \in \mathbb{N}}\left|s^{2} x_{k-2}+2 r s x_{k-1}+r^{2} x_{k}\right|<\infty\right\}, \\
c(\widehat{\widehat{B}}):=\left\{x=\left(x_{k}\right) \in w: \exists l \in \mathbb{C} \ni \lim _{k \rightarrow \infty}\left|s^{2} x_{k-2}+2 r s x_{k-1}+r^{2} x_{k}-l\right|=0\right\}, \\
c_{0}(\widehat{\widehat{B}}):=\left\{x=\left(x_{k}\right) \in w: \lim _{k \rightarrow \infty}\left|s^{2} x_{k-2}+2 r s x_{k-1}+r^{2} x_{k}\right|=0\right\}, \\
\ell_{p}(\widehat{\widehat{B}}):=\left\{x=\left(x_{k}\right) \in w:\left.\sum_{k}\left|s^{2} x_{k-2}+2 r s x_{k-1}+r^{2} x_{k}\right|\right|^{p}<\infty\right\} .
\end{gathered}
$$

In other words; the sets defined above; of all sequences whose $\widehat{\widehat{B}}$-transforms are in the spaces $\ell_{\infty}, c, c_{0}$ and $\ell_{p}$, respectively. Considering the matrix domain with the notation of (1.2), the remarkable feature of these sets is as follows $\ell_{\infty}(\widehat{\widehat{B}}), c(\widehat{\widehat{B}})$, $c_{0}(\widehat{\widehat{B}})$ and $\ell_{p}(\widehat{\widehat{B}})$ by $\ell_{\infty}(\widehat{\widehat{B}}):=\left\{\ell_{\infty}\right\}_{\widehat{B}}, c(\widehat{\widehat{B}}):=c_{\widehat{\widehat{B}}}, c_{0}(\widehat{\widehat{B}}):=\left\{c_{0}\right\}_{\widehat{B}}$ and $\ell_{p}(\widehat{\widehat{B}}):=\left\{\ell_{p}\right\}_{\widehat{\widehat{B}}}$. When $x=\left(x_{k}\right)$ is a sequence and the transformation $\widehat{\widehat{B}}$ of $x=\left(x_{k}\right)$ which is defined by matrix multiplication is the sequence $y=\left(y_{k}\right)$, we shall write

$$
y_{k}:=s^{2} x_{k-2}+2 r s x_{k-1}+r^{2} x_{k}, \quad(k \in \mathbb{N}) .
$$

Before beginning the general theory, at first we should state the following fundamental theorem, showing that sets just described have an important role in their algebraic structures.

Theorem 2.1. $\ell_{\infty}(\widehat{\widehat{B}}), c(\widehat{\widehat{B}}), c_{0}(\widehat{\widehat{B}})$ and $\ell_{p}(\widehat{\widehat{B}})$ are sets which are linear spaces given by coordinatewise addition and also scalar multiplication, that is, those $\ell_{\infty}(\widehat{\widehat{B}}), c(\widehat{\widehat{B}}), c_{0}(\widehat{\widehat{B}})$ and $\ell_{p}(\widehat{\widehat{B}})$ sets are in fact the sequence spaces.

Proof. Since the calculations involving coordinatewise addition and scalar multiplication are considerably simply, details of the proof will not be given here.

Now it is time to give the definition of isomorphism between two linear spaces. Let $U$ and $V$ be linear spaces. We say that $U$ is isomorphic to $V$ if there exists a linear transformation $T: U \rightarrow V$ that is invertible. Such a linear transformation is called an isomorphism from $U$ onto $V$ and it is written $U \approx V$.

We now derive one of the most important properties of $X \in\left\{\ell_{\infty}, c, c_{0}, \ell_{p}\right\}$ and $X_{\widehat{B}}$ which are used extensively.

Theorem 2.2. The newly defined sequence spaces $\ell_{\infty}(\widehat{\widehat{B}}), c(\widehat{\widehat{B}}), c_{0}(\widehat{\widehat{B}})$ and $\ell_{p}(\widehat{\widehat{B}})$ are norm isomorphic to the classical sequence spaces $\ell_{\infty}, c, c_{0}$ and $\ell_{p}$, respectively; that is, $\ell_{\infty}(\widehat{\widehat{B}}) \approx \ell_{\infty}, c(\widehat{\widehat{B}}) \approx c, c_{0}(\widehat{\widehat{B}}) \approx c_{0}$ and $\ell_{p}(\widehat{\widehat{B}}) \approx \ell_{p}$.

Proof. When focusing on the proof, it is almost the same to show that the newly defined sequence spaces related to the classical sequence spaces are linear isomorphs, so we are going to prove only one here. What is needed to verify this allegation is to guarantee the existence by the technique used in solving previously published papers could also have been used in from the $c_{0}(\widehat{\widehat{B}})$ and $c_{0}$. When the transformation $T$ described above is taken into consideration again, taking $y=T(x)=\widehat{\widehat{B}} x$ in the definition of $T$, we can see that $T$ is a linear transformation between $c_{0}(\widehat{\widehat{B}})$ to $c_{0}$ when (1.2) is used. We shall not prove it in detail. Over and above, it is obtained $x=\theta$ from some basic calculations whenever $T(x)=\theta$. It follows from this fact that $T$ is injective. Thus, we choose an $y=\left(y_{k}\right) \in c_{0}$ and inverse transformation provides us the possibility to identify the sequence $x=\left(x_{k}\right)$ by

$$
x_{k}:=\frac{1}{r^{2}} \sum_{j=0}^{k}(k-j+1)\left(-\frac{s}{r}\right)^{k-j} y_{j} \quad(k \in \mathbb{N})
$$


then from (2.1) we successively calculate

$$
\begin{array}{r}
s^{2} x_{k-2}+2 r s x_{k-1}+r^{2} x_{k}=s^{2}\left[\frac{1}{r^{2}} \sum_{j=0}^{k-2}(k-j-1)\left(-\frac{s}{r}\right)^{k-j-2} y_{j}\right] \\
+2 r s\left[\frac{1}{r^{2}} \sum_{j=0}^{k-1}(k-j)\left(-\frac{s}{r}\right)^{k-j-1} y_{j}\right]+r^{2}\left[\frac{1}{r^{2}} \sum_{j=0}^{k}(k-j+1)\left(-\frac{s}{r}\right)^{k-j} y_{j}\right]=y_{k}
\end{array}
$$

for every $k \in \mathbb{N}$.

From the last calculations, we conclude that the $x=\left(x_{k}\right)$ defined by above lies $c_{0}(\widehat{\widehat{B}})$ since the $y=\left(y_{k}\right)$ lies $c_{0}$. This means that $T$ is surjective. All that we have done so far is to show that the newly defined space $c_{0}(\widehat{\widehat{B}})$ and well-known space $c_{0}$ are linearly isomorphic. We accomplished the proof by carrying out the necessary steps.

Theorem 2.3. Let $X \in\left\{\ell_{\infty}, c, c_{0}, \ell_{p}\right\}$ and the matrix $\widehat{\widehat{B}}$ defined above. Then,

(i) $X=X(\widehat{\widehat{B}})$ if $\left|\frac{s}{r}\right|<1$.

(ii) $X \subset X(\widehat{\widehat{B}})$ is strict if $\left|\frac{s}{r}\right| \geq 1$.

Proof. Let $X \in\left\{\ell_{\infty}, c, c_{0}, \ell_{p}\right\}$. The usage of appropriate properties of maths leads to obtaining equations for the matrix $\widehat{\widehat{B}}$ that are briefly written below but actually require long calculations.

$$
\begin{gathered}
\sup _{n \in \mathbb{N}} \sum_{k}\left|\widehat{\widehat{b}}_{n k}\right|=(|r|+|s|)^{2}, \quad \lim _{n \rightarrow \infty} \widehat{\widehat{b}}_{n k}=0, \\
\lim _{n \rightarrow \infty} \sum_{k} \widehat{\widehat{b}}_{n k}=(r+s)^{2} \text { and } \sup _{k \in \mathbb{N}} \sum_{n}\left|\widehat{\widehat{b}}_{n k}\right|=(|r|+|s|)^{2},
\end{gathered}
$$

$\widehat{\widehat{B}} \in(X: X)$. Because of the above explanation, we get $x \in X(\widehat{\widehat{B}})$ for any sequence $x \in X$. when these facts are used, we see that the inclusion $X \subset X(\widehat{\widehat{B}})$ is hold.

(i) We consider first the case where $\left|\frac{s}{r}\right|<1$. In proving that the following conditions are met for inverse matrix $\widehat{\widehat{B}}^{-1}:=\left(\widehat{\widehat{b}}_{n k}^{-1}\right)$ of the matrix $\widehat{\widehat{B}}$, we follow a similar way to the above.

$$
\begin{aligned}
\sup _{n \in \mathbb{N}} \sum_{k}\left|\widehat{\widehat{b}}_{n k}^{-1}\right| & =\frac{1}{r^{2}} \sum_{k}(k+1)\left|\frac{s}{r}\right|^{k}<\infty, \\
\lim _{n \rightarrow \infty} \widehat{\widehat{b}}_{n k}^{-1} & =\lim _{n \rightarrow \infty}(n-k+1)\left(-\frac{s}{r}\right)^{n-k} \\
& =\left(-\frac{s}{r}\right)^{-k} \lim _{n \rightarrow \infty}(n-k)\left(-\frac{s}{r}\right)^{n} \\
& =0, \\
\lim _{n \rightarrow \infty} \sum_{k} \widehat{\widehat{b}}_{n k}^{-1}= & \frac{1}{r^{2}} \lim _{n \rightarrow \infty} \sum_{k=1}^{n} k\left(-\frac{s}{r}\right)^{k-1} \text { exists, } \\
\sup _{k \in \mathbb{N}} \sum_{n}\left|\widehat{\widehat{b}}_{n k}^{-1}\right| & =\frac{1}{r^{2}} \sum_{n}(n+1)\left|\frac{s}{r}\right|^{n}<\infty,
\end{aligned}
$$

$\widehat{\widehat{B}}^{-1} \in(X: X)$, where

$$
\widehat{b}_{n k}^{-1}:=\left\{\begin{array}{cl}
(n-k+1) \frac{1}{r^{2}}\left(-\frac{s}{r}\right)^{n-k} & , \quad(0 \leq k \leq n), \\
0 & , \quad(k>n)
\end{array}\right.
$$

for every $k, n \in \mathbb{N}$. We know that $x \in X(\widehat{\widehat{B}})$ and when we apply the definition, we get $y=\widehat{\widehat{B}} x \in X$ and the next step makes the fact that $x=\widehat{\widehat{B}}^{-1} y \in X$. This means that the inclusion $X(\widehat{\widehat{B}}) \subset X$ is fulfilled. This shows that the proof of Part (i) is over. 
(ii) When getting results we need to use the following.

$$
\begin{aligned}
t^{1} & :=\left\{\frac{n+1}{r^{2}}\left(-\frac{s}{r}\right)^{n}\right\} \\
t^{2} & :=\left\{\frac{n+1}{r^{2}}\right\}, t^{3}:=\left\{(-1)^{n}(n+2)\right\} \text { and } t^{4}:=\left\{\frac{1+(-1)^{n}}{2}\right\} .
\end{aligned}
$$

With a simple approximation, if $\left|\frac{s}{r}\right|>1$ holds, then $\widehat{\widehat{B}} t^{1}=e^{(0)}=(1,0,0, \ldots, 0, \ldots) \in X$. Thus, we have $t^{1} \in X(\widehat{\widehat{B}})$. But, the sequence $t^{1}$ is unbounded and then $t^{1} \in X(\widehat{\widehat{B}}) \backslash X$.

Suppose that $\left|\frac{s}{r}\right|=1$.

(a) Let $X=c_{0}, \ell_{p}$. Then, $t^{1} \in X(\widehat{\widehat{B}}) \backslash X$.

(b) Let $X=\ell_{\infty}, c$. Then,

i) When $s=-r$ is taken, the transformation of $t^{2}$ is $\widehat{\widehat{B}} t^{2}=e^{(0)}=(1,0,0, \ldots, 0, \ldots) \in X$. Hence $t^{2} \in X(\widehat{\widehat{B}}) \backslash X$.

ii) If $s=r$, then $\widehat{\widehat{B}} t^{3}=\left(2 r^{2}, r^{2}, 0, \ldots, 0, \ldots\right) \in \ell_{\infty}$ and

$\widehat{\widehat{B}} t^{4}=\left(r^{2}, 2 r^{2}, 2 r^{2}, \ldots, 2 r^{2}, \ldots\right) \in c$. Hence $t^{3} \in \ell_{\infty}(\widehat{\widehat{B}}) \backslash \ell_{\infty}$ and $t^{4} \in c(\widehat{\widehat{B}}) \backslash c$.

Clearly, from of these, we have precisely shown that the inclusion $X \subset X_{\widehat{\widehat{B}}}$ is strict.

We have the following terminology used by almost everyone studying in this field. Let $X$ and $Y$ arbitrary two sequence spaces. Known as the multiplier space $S(X, Y)$ defined by

$$
S(X, Y):=\left\{t=\left(t_{k}\right) \in w: x t=\left(x_{k} t_{k}\right) \in Y \text { for all } x=\left(x_{k}\right) \in X\right\}
$$

is set. We now look at some additional properties of multiplier space. Let $X, Y$ and $Z$ denote any three sequence spaces and $X \supset Z \supset Y$. In view of the preceding elementary knowledge, we see that the inclusions $S(X, Y) \subset S(Z, Y)$ and also $S(X, Y) \subset S(X, Z)$. The notation of (2.2) provides an easy of forming the duals as follows $X^{\alpha}, X^{\beta}$ and $X^{\gamma}$ are defined by $X^{\alpha}=S\left(X, \ell_{1}\right), X^{\beta}=S(X, c s)$ and $X^{\gamma}=S(X, b s)$.

Lemma 2.4. Let $X, Y$ be the sequence spaces and $\xi \in\{\alpha, \beta, \gamma\}$. If $X \subset Y$ then $Y^{\xi} \subset X^{\xi}$.

The following list helps in dealing with some difficult situations:

$$
\begin{gathered}
\sup _{n \in \mathbb{N}} \sum_{k}\left|a_{n k}\right|^{q}<\infty \\
\sup _{k, n \in \mathbb{N}}\left|a_{n k}\right|<\infty \\
\lim _{n \rightarrow \infty} a_{n k}=a_{k} \text { for each } k \in \mathbb{N} \\
\lim _{n \rightarrow \infty} \sum_{k}\left|a_{n k}\right|=\sum_{k}\left|a_{k}\right| \\
\lim _{n \rightarrow \infty} \sum_{k} a_{n k}=a
\end{gathered}
$$

The following lemma presented by Stieglitz and Tietz [25] is particularly useful in obtaining that certain properties.

Lemma 2.5. The necessary and sufficient conditions for $A \in(X: Y)$ when $X \in\left\{\ell_{\infty}, c, c_{0}, \ell_{p}, \ell_{1}\right\}$ and $Y \in\left\{\ell_{\infty}, c\right\}$ can be read from Table 1, where
1. (2.3) with $q=1$.
2. (2.3).
3. (2.4).
4. (2.5) and (2.6).
5. (2.3) with $q=1,(2.5)$ and (2.7).
6. (2.3) with $q=1$ and (2.5).
7. (2.3) and (2.5).
8. (2.4) and (2.5).

Table 1: The characterization of the class $(X, Y)$ with $X \in\left\{\ell_{\infty}, c, c_{0}, \ell_{p}, \ell_{1}\right\}$ and $Y \in\left\{\ell_{\infty}, c\right\}$

\begin{tabular}{cccccc}
\hline From & $\ell_{\infty}$ & $c$ & $c_{0}$ & $\ell_{p}$ & $\ell_{1}$ \\
\hline To & & & & & \\
\hline$\ell_{\infty}$ & $\mathbf{1 .}$ & $\mathbf{1 .}$ & $\mathbf{1 .}$ & $\mathbf{2 .}$ & $\mathbf{3 .}$ \\
$c$ & $\mathbf{4 .}$ & $\mathbf{5 .}$ & $\mathbf{6 .}$ & $\mathbf{7 .}$ & $\mathbf{8 .}$ \\
\hline
\end{tabular}


Lemma 2.6. ([8, Theorem 3.1]) Let $C=\left(c_{n k}\right)$ be defined via a sequence $b=\left(b_{k}\right) \in w$ and inverse matrix $D=\left(d_{n k}\right)$ of triangle matrix $U=\left(u_{n k}\right)$ by

$$
c_{n k}:=\left\{\begin{array}{cl}
\sum_{j=k}^{n} b_{j} d_{j k} & , \quad(0 \leq k \leq n), \\
0 & , \quad(k>n),
\end{array}\right.
$$

for all $k, n \in \mathbb{N}$. Then,

$$
\begin{gathered}
\left\{X_{U}\right\}^{\gamma}:=\left\{b=\left(b_{k}\right) \in w: C \in\left(X: \ell_{\infty}\right)\right\}, \\
\left\{X_{U}\right\}^{\beta}:=\left\{b=\left(b_{k}\right) \in w: C \in(X: c)\right\} .
\end{gathered}
$$

When both Lemma 2.5 and Lemma 2.6 are considered together, it is seen that the following corollary will be obtained.

Corollary 2.7. Define the sets $\widehat{\hat{d}_{1}}, \widehat{\hat{d}_{2}}, \widehat{\hat{d}_{3}}, \widehat{\widehat{d}}_{4}$ and $\widehat{\widehat{d}}_{5}$ by

$$
\begin{aligned}
& \widehat{\hat{d}_{1}}=\left\{a=\left(a_{k}\right) \in w: \sup _{n \in \mathbb{N}} \sum_{k=0}^{n}\left|\frac{1}{r^{2}} \sum_{j=k}^{n}(j-k+1)\left(-\frac{s}{r}\right)^{j-k} a_{j}\right|^{q}<\infty\right\}, \\
& \widehat{\widehat{d}_{2}}=\left\{a=\left(a_{k}\right) \in w: \lim _{n \rightarrow \infty} \frac{1}{r^{2}} \sum_{j=k}^{n}(j-k+1)\left(-\frac{s}{r}\right)^{j-k} a_{j} \text { exist }\right\} \\
& \widehat{\widehat{d}_{3}}=\left\{a=\left(a_{k}\right) \in w: \lim _{n \rightarrow \infty} \sum_{k=0}^{n}\left|\sum_{j=k}^{n} b_{j k}^{-1} a_{j}\right|=\sum_{k=0}^{\infty}\left|\lim _{n \rightarrow \infty} \frac{1}{r^{2}} \sum_{j=k}^{n}(j-k+1)\left(-\frac{s}{r}\right)^{j-k} a_{j}\right|\right\}, \\
& \widehat{\hat{d}_{4}}=\left\{a=\left(a_{k}\right) \in w: \lim _{n \rightarrow \infty} \frac{1}{r^{2}} \sum_{k=0}^{n} \sum_{j=k}^{n}(j-k+1)\left(-\frac{s}{r}\right)^{j-k} a_{j} \text { exist }\right\} \\
& \widehat{\widehat{d}_{5}}=\left\{a=\left(a_{k}\right) \in w: \sup _{n, k \in \mathbb{N}} \frac{1}{r^{2}}\left|\sum_{j=k}^{n}(j-k+1)\left(-\frac{s}{r}\right)^{j-k} a_{j}\right|<\infty\right\},
\end{aligned}
$$

Then,

(i) $\left\{\ell_{\infty}(\widehat{\widehat{B}})\right\}^{\gamma}=c(\widehat{\widehat{B}})^{\gamma}=\left\{c_{0}(\widehat{\widehat{B}})\right\}^{\gamma}:=\widehat{\widehat{d}}_{1}$ with $q=1$.

(ii) $\left\{\ell_{p}(\widehat{\widehat{B}})\right\}^{\gamma}:=\widehat{\widehat{d}}_{1}$.

(iii) $\left\{\ell_{1}(\widehat{\widehat{B}})\right\}^{\gamma}:=\widehat{\widehat{d}}_{5}$.

(iv) $\left\{c_{0}(\widehat{\widehat{B}})\right\}^{\beta}:=\widehat{\widehat{d}}_{1} \cap \widehat{\widehat{d}}_{2}$ with $q=1$.

(v) $\{c(\widehat{\widehat{B}})\}^{\beta}:=\widehat{\widehat{d}}_{1} \cap \widehat{\widehat{d}}_{2} \cap \widehat{\widehat{d}}_{4}$ with $q=1$.

(vi) $\left\{\ell_{p}(\widehat{\widehat{B}})\right\}^{\beta}:=\widehat{\widehat{d}}_{1} \cap \widehat{\widehat{d}}_{2}$.

(vii) $\left\{\ell_{1}(\widehat{\widehat{B}})\right\}^{\beta}:=\widehat{\widehat{d}}_{2} \cap \widehat{\widehat{d}}_{5}$.

(viii) $\left\{\ell_{\infty}(\widehat{\widehat{B}})\right\}^{\beta}:=\widehat{\widehat{d}}_{2} \cap \widehat{\widehat{d}}_{3}$.

Sequence space $X$ having a linear topology is known as $K$-space when every map $p_{i}: X \rightarrow \mathbb{C}$ described by $p_{i}(x)=x_{i}$ is continuous for every $i \in \mathbb{N}$. Again $A K$-space $X$ is known as $F K$ - space when $X$ satisfies the condition of being a complete linear metric space. When $F K$-space of which topology is known as $B K$-space. When a normed sequence space $X$ including a sequence $\left(b_{n}\right)$ having the characteristics of having a unique sequence of scalars $\left(a_{n}\right)$ for each $x \in X$ such that

$$
\lim _{n \rightarrow \infty}\left\|x-\left(\alpha_{0} b_{0}+\alpha_{1} b_{1}+\cdots+\alpha_{n} b_{n}\right)\right\|=0,
$$

under this condition $\left(b_{n}\right)$ is known as Schauder basis for $X$ (or in short form only basis). Then the series $\sum a_{k} b_{k}$ having the summation $x$ is known as the expansion of $x$ in terms of $\left(b_{n}\right)$, and denoted by $x=\sum a_{k} b_{k}$. Because of the fact that, the matrix domain $X_{A}$ of a normed sequence space denoted by $X$ has got a basis iff $X$ has got a basis when $A=\left(a_{n k}\right)$ is a triangle, one can obtain: 
Corollary 2.8. Let $\alpha_{k}(r)=\{\widehat{\widehat{B}} x\}_{k}$ for all $k \in \mathbb{N}$. Define the sequence $z=\left(z_{k}\right)$ and $\widehat{\widehat{\hat{b}}}^{(k)}=\left\{\widehat{\hat{b}}^{(k)}\right\}_{n \in \mathbb{N}}$ for every fixed $k \in \mathbb{N}$ by $z_{n}=\sum_{k=0}^{n} \widehat{\widehat{b}}_{n k}^{-1}$ and $\widehat{\widehat{b}}_{n}^{(k)}=\left\{\begin{array}{ccc}0 & (n<k), \\ \widehat{\widehat{b}}_{n k}^{-1}, & (n \geq k) .\end{array}\right.$ Then,

(a) The sequence $\left\{\widehat{\widehat{b}}^{(k)}\right\}_{n \in \mathbb{N}}$ is a basis for the spaces $c_{0}(\widehat{\widehat{B}})$ and $\ell_{p}(\widehat{\widehat{B}})$, and any $x$ in $c_{0}(\widehat{\widehat{B}})$ or in $\ell_{p}(\widehat{\widehat{B}})$ has a unique representation of the form

$$
x:=\sum_{k} \alpha_{k}(r) \widehat{\widehat{b}}^{(k)} .
$$

(b) The set $\left\{z, \widehat{\widehat{b}}^{(k)}\right\}_{n \in \mathbb{N}}$ is a basis for the spaces $c(\widehat{\widehat{B}})$ and any $x$ in $c(\widehat{\widehat{B}})$ has a unique representation of the form

$$
x:=l z+\sum_{k}\left[\alpha_{k}(r)-l\right] \widehat{\widehat{b}}^{(k)},
$$

where $l=\lim _{k \rightarrow \infty}\{\widehat{\widehat{B}} x\}_{k}$.

It is known that the $X Y$ set means

$$
X Y:=\left\{z=\left(z_{k}\right) \in w: z_{k}=x_{k} y_{k}, \forall k \in \mathbb{N}, x=\left(x_{k}\right) \in X, y=\left(y_{k}\right) \in Y\right\}
$$

for the sequence spaces $X$ and $Y$. When a $B K$-space $X \supset \phi$ is given, the $\mathrm{n} t h$ section $x^{[n]}$ of the sequence $x=\left(x_{k}\right) \in X$ is described by $x^{[n]}:=\sum_{k=0}^{n} x_{k} e^{(k)}$ and it is said that $x$ has got the characteristics:

$A K$ if $\lim _{n \rightarrow \infty}\left\|x-x^{[n]}\right\|_{X}=0$ (abschnittskonvergenz),

$A B$ if $\sup _{n \in \mathbb{N}}\left\|x^{[n]}\right\|_{X}<\infty$ (abschnittsbeschränktheit),

$A D$ if $x \in \phi$ (clousure of $\phi \subset X)$ (abschnittsdichte),

$K B$ if the set $\left\{x_{k} e^{(k)}\right\}$ is bounded in $X$ (koordinatenweise beschränkt).

It is said that the space $X$ has a property if this property is held for each $x \in X$, (cf. [26]). One can obviously see that $A K$ implies $A D$ and $A K$ if and only if $A B+A D$. To give an example for this fact, even though $c_{0}$ and $\ell_{p}$ are $A K$-spaces, $c$ and $\ell_{\infty}$ are not $A D$-spaces.

Lemma 2.9. ([27, Theorem 2.1 and Lemma 4.1]). Let $X, Y$ be the BK-spaces and $F_{Y}^{U}=\left(f_{n k}\right)$ be defined via the sequence $\alpha=\left(\alpha_{k}\right) \in Y$ and the triangle matrix $U=\left(u_{n k}\right)$ by

$$
f_{n k}=\sum_{j=k}^{n} \alpha_{j} u_{n j} d_{j k}
$$

for all $k, n \in \mathbb{N}$. Then, the domain of the matrix $U$ in the sequence space $X$ has the following properties

(i) $K B$ iff $F_{\ell_{1}}^{U} \in(X: X)$.

(ii) $A B$ iff $F_{b_{v_{0}}}^{U} \in(X: X)$.

From Lemma 2.9, we have:

Corollary 2.10. Let $\left|\frac{s}{r}\right|$ be equal to 1 . Under this condition, the space $\ell_{1}(\widehat{\widehat{B}})$ has both the KB-and AB-properties.

Lemma 2.11. [27, Theorem 2.2] Let $X$ be a BK-space which has AK-property, $U$ be a triangle matrix and $X_{U} \supset \phi$. Then the sequence space $X_{U}$ has the $A D$-property if and only if the fact $t U=\theta$ for $t \in X^{\beta}$ implies the fact $t=\theta$.

We know that both $c_{0}$ and $\ell_{p}$ have the $A K$-property, when $U=\widehat{\widehat{B}}$, we get the following corollary by applying Lemma 2.11 .

Corollary 2.12. $c_{0}(\widehat{\widehat{B}})$ and $\ell_{p}(\widehat{\widehat{B}})(p>1)$ have the AD-property if and only if $\left|\frac{s}{r}\right| \leq 1$.

3. Some matrix transformations related to the sequence spaces $\ell_{\infty}(\widehat{\widehat{B}}), c(\widehat{\widehat{B}}), c_{0}(\widehat{\widehat{B}})$ and $\ell_{1}(\widehat{\widehat{B}})$

The present section is devoted to the characterization of some classes of infinite matrices related with newly defined sequence spaces. The following theorem about matrix transformations is analogous to the corresponding theorem obtained by the previous ones. It tells us how to combine those results as necessary and sufficient condition. 
Theorem 3.1. Let us assume that $X$ be an $F K$-space, $U$ be a triangle matrix, $D$ denotes its inverse matrix and $Y$ be any subset of $\omega$. Under these assumptions, one conclude that $A=\left(a_{n k}\right) \in\left(X_{U}: Y\right)$ iff

$$
C^{(n)}:=\left(c_{m k}^{(n)}\right) \in(X: c) \text { for all } n \in \mathbb{N}
$$

and

$$
C=\left(c_{n k}\right) \in(X: Y)
$$

in which

$$
c_{m k}^{(n)}:=\left\{\begin{array}{cll}
\sum_{j=k}^{m} a_{n j} d_{j k} & , \quad(0 \leq k \leq m), \\
0 & , \quad(k>m),
\end{array} \quad \text { and } c_{n k}:=\sum_{j=k}^{\infty} a_{n j} d_{j k}\right.
$$

for every $k, m, n \in \mathbb{N}$.

Proof. Suppose that $A=\left(a_{n k}\right) \in\left(X_{U}: Y\right)$ and let us take $x \in X_{U}$. Under these assumptions, we leave it to the reader to verify that following equations are indeed satisfied

$$
\sum_{k=0}^{m} a_{n k} x_{k}=\sum_{k=0}^{m} a_{n k}\left(\sum_{j=0}^{k} d_{k j} y_{j}\right)=\sum_{k=0}^{m}\left(\sum_{j=k}^{m} a_{n j} d_{j k}\right) y_{k}=\sum_{k=0}^{m} c_{m k}^{(n)} y_{k}
$$

for all $m, n \in \mathbb{N}$. Due to the fact that $A x$ exists, we deduce that $C^{(n)}$ must belong to the class $(X: c)$. By passing to limit $m \rightarrow \infty$ in the equality (3.3) we can easily deduce $A x=C y$. It is obtained that $C y \in Y$, using $A x \in Y$. This means that $C \in(X: Y)$. In a converse way, let us assume that (3.1), (3.2) are met and let us consider any $x \in X_{U}$. In these conditions, we take $\left(c_{n k}\right)_{k \in \mathbb{N}} \in X^{\beta}$ it is obtained that $\left(a_{n k}\right)_{k \in \mathbb{N}} \in X_{U}^{\beta}$ for all $n \in \mathbb{N}$, using (3.1). This tells us the existence of the $A$-transform of $x$, namely $A x$ exists. Moreover, we derive from the equality (3.3) as $m \rightarrow \infty$ that $A x=C y$ and this indicates that $A \in\left(X_{U}: Y\right)$.

Now, we list the following conditions:

$$
\begin{gathered}
\sup _{m \in \mathbb{N}} \sum_{k=0}^{m}\left|\frac{1}{r^{2}} \sum_{j=k}^{m}(j-k+1)\left(-\frac{s}{r}\right)^{j-k} a_{n j}\right|^{q}<\infty \\
\lim _{m \rightarrow \infty} \frac{1}{r^{2}} \sum_{j=k}^{m}(j-k+1)\left(-\frac{s}{r}\right)^{j-k} a_{n j}=c_{n k} \\
\lim _{m \rightarrow \infty} \sum_{k=0}^{m}\left|\frac{1}{r^{2}} \sum_{j=k}^{m}(j-k+1)\left(-\frac{s}{r}\right)^{j-k} a_{n j}\right|=\sum_{k}\left|c_{n k}\right| \text { for each } n \in \mathbb{N} \\
\lim _{m \rightarrow \infty} \sum_{k=0}^{m} \frac{1}{r^{2}} \sum_{j=0}^{k}(j-k+1)\left(-\frac{s}{r}\right)^{j-k} a_{n k}=\alpha_{n} \text { for all } n \in \mathbb{N} \\
\sup _{m, k \in \mathbb{N}}\left|\frac{1}{r^{2}} \sum_{j=k}^{m}(j-k+1)\left(-\frac{s}{r}\right)^{j-k} a_{n j}\right|<\infty \\
\sup _{n \in \mathbb{N}} \sum_{k}\left|c_{n k}\right|^{q}<\infty \\
\lim _{n \rightarrow \infty} c_{n k}=\beta_{k} \\
\lim _{n \rightarrow \infty} \sum_{k}\left|c_{n k}\right|=\sum_{k}\left|\beta_{k}\right| \\
\lim _{n \rightarrow \infty} \sum_{k} c_{n k}=\beta
\end{gathered}
$$




$$
\begin{gathered}
\sup _{n, k \in \mathbb{N}}\left|c_{n k}\right|<\infty \\
\sup _{k \in \mathbb{N}} \sum_{n}\left|c_{n k}\right|<\infty \\
\lim _{n \rightarrow \infty} \sum_{k} c_{n k}=0 \\
\sup _{N, K \in \mathscr{F}}\left|\sum_{n \in N} \sum_{k \in K} c_{n k}\right|<\infty \\
\sup _{N \in \mathscr{F}} \sum_{k}\left|\sum_{n \in N} c_{n k}\right|^{q}<\infty,
\end{gathered}
$$

in which the symbol $\mathscr{F}$ illustrates the collection of all finite subsets of $\mathbb{N}$. We note here that, Theorem 3.1 tells us that we will have the following table.

Table 2. The characterization of the class $A \in(X: Y)$ with $X \in\left\{\ell_{\infty}(\widehat{\widehat{B}}), c(\widehat{\widehat{B}}), c_{0}(\widehat{\widehat{B}}), \ell_{p}(\widehat{\widehat{B}})\right\}$ and $Y \in\left\{\ell_{\infty}, c, c_{0}, \ell_{1}\right\}$.

\begin{tabular}{cccccc}
\hline From & $\ell_{\infty}(\widehat{\widehat{B}})$ & $c(\widehat{\widehat{B}})$ & $c_{0}(\widehat{\widehat{B}})$ & $\ell_{p}(\widehat{\widehat{B}})$ & $\ell_{1}(\widehat{\widehat{B}})$ \\
\hline To & & & & & \\
\hline$\ell_{\infty}$ & $\mathbf{1 .}$ & $\mathbf{2 .}$ & $\mathbf{3 .}$ & $\mathbf{4 .}$ & $\mathbf{5 .}$ \\
$c$ & $\mathbf{6 .}$ & $\mathbf{7 .}$ & $\mathbf{8 .}$ & $\mathbf{9 .}$ & $\mathbf{1 0 .}$ \\
$c_{0}$ & $\mathbf{1 1 .}$ & $\mathbf{1 2 .}$ & $\mathbf{1 3 .}$ & $\mathbf{1 4 .}$ & $\mathbf{1 5 .}$ \\
$\ell_{1}$ & $\mathbf{1 6 .}$ & $\mathbf{1 7 .}$ & $\mathbf{1 8 .}$ & $\mathbf{1 9 .}$ & $\mathbf{2 0 .}$ \\
\hline
\end{tabular}

Corollary 3.2. The necessary and sufficient conditions for all $A \in(X: Y)$ when $X \in\left\{\ell_{\infty}(\widehat{\widehat{B}}), c(\widehat{\widehat{B}}), c_{0}(\widehat{\widehat{B}}), \ell_{p}(\widehat{\widehat{B}})\right\}$ and $Y \in$ $\left\{\ell_{\infty}, c, c_{0}, \ell_{1}\right\}$ can be read from the Table 2 : where,

1. (3.5), (3.6) and (3.9) with $q=1$.

2. (3.5), (3.7) and (3.4),(3.9) with $q=1$.

3. (3.5) and (3.4), (3.9) with $q=1$.

4. (3.4), (3.5) and (3.9).

5. (3.5), (3.8) and (3.13).

6. (3.5), (3.6), (3.10) and (3.11).

7. (3.5), (3.7), (3.10), (3.12) and (3.4), (3.9) with $q=1$.

8. (3.5), (3.10) and (3.4), (3.9) with $q=1$.

9. (3.4), (3.5), (3.9) and (3.10).

10. (3.5), (3.8), (3.10) and (3.13).

11. (3.5), (3.6) and (3.15).

12. (3.5), (3.7), (3.10) with $\beta_{k}=0$ and(3.12) with $\beta=0$ and (3.4), (3.9) with $q=1$.

13. (3.5), (3.10) with $\beta_{k}=0$ and (3.4), (3.9) with $q=1$.

14. (3.4), (3.5), (3.9) and (3.10) with $\beta_{k}=0$.

15. (3.5), (3.8), (3.10) with $\beta_{k}=0$ and (3.13).

16. (3.5), (3.6) and (3.16).

17. (3.4) with $q=1,(3.5),(3.7)$ and (3.16).

18. (3.4) with $q=1,(3.5)$ and (3.16).

19. (3.4), (3.5) and (3.17).

20. (3.5), (3.8) and (3.14).

Now, we are going to present the following lemma leading more quickly to the computation of the characterization of some new matrix classes, using the Corollary 3.2.

Lemma 3.3. [28, Lemma 5.3] Let $X, Y$ be arbitrary two sequence spaces, $A$ be an infinite matrix and $U$ a triangle matrix. Then, $A \in\left(X: Y_{U}\right)$ iff $U A \in(X: Y)$.

Here we are able to give an ultimate note. When based on writing $r^{2} a_{n k}+2 r s a_{n-1, k}+s^{2} a_{n-2, k}$ instead of $a_{n k}$ for all $k, n \in \mathbb{N}$ in Corollary 3.2 , since $U=\widehat{\widehat{B}}$ is triangle matrix, we can actually find out the characterization of the class $(X(\widehat{\widehat{B}}): Y(\widehat{\widehat{B}}))$ using Lemma 3.3. 


\section{Conclusion}

In brief, the present manuscript has investigated algebraic and topological characteristics of the sequence space $X(\widehat{\widehat{B}}(r, s))$. The $\beta$ - and $\gamma$-duals for these spaces have been calculated at the same time Schauder bases for those spaces $c(\widehat{\widehat{B}}), c_{0}(\widehat{\widehat{B}})$ and $\ell_{p}(\widehat{\widehat{B}})$ are found out. It has been noted that all of these spaces have got special characteristics. Some matrix transformations have been characterized.

\section{Acknowledgements}

Some results of this work were partially presented at the $18^{\text {th }}$ International Geometry Symposium to be held on $12-13$ July, 2021 in Malatya, TURKEY at İNÖNÜ UNIVERSITY.

\section{Funding}

There is no funding for this work.

\section{Availability of data and materials}

Not applicable.

\section{Competing interests}

The authors declare that they have no competing interests.

\section{Author's contributions}

All authors contributed equally to the writing of this paper. All authors read and approved the final manuscript.

\section{References}

[1] F. Başar, Summability Theory and Its Applications, Bentham Science Publishers, İstanbul, 2012, ISBN: 978-1-60805-252-3.

[2] F. Başar \& H. Dutta, Summable Spaces and Their Duals, Matrix Transformations and Geometric Properties, CRC Press, Taylor \& Francis Group, Monographs and Research Notes in Mathematics, Boca Raton • London - New York, 2020. ISBN: 978-0-8153-5177-1.

[3] M. Mursaleen, F. Başar, Sequence Spaces: Topics in Modern Summability Theory, CRC Press, Taylor \& Francis Group, Series: Mathematics and Its Applications, Boca Raton · London • New York, 2020.

[4] M. Mursaleen, Applied Summability Methods, Springer Briefs, 2014.

[5] B. de Malafosse, E.Malkowsky, and V. Rakocevic, Operators Between Sequence Spaces and Applications, Springer Nature Singapore, 152 Beach Road, Singapore 18972, Singapore.

[6] H. Kızmaz, On certain sequence spaces, Canad. Math. Bull. 24(2) (1981), 169-176.

[7] M. Et, On some difference sequence spaces, Turk. J. Math. 17 (1993), 18-24.

[8] M. A. Sarıö̈l, On difference sequence spaces, J. Karadeniz Tech. Uni. Fac. Arts Sci. Ser. Math.-Phys., 10 (1987), 63-71.

[9] Z. U. Ahmad, M. Mursaleen, Köthe-Toeplitz duals of some new sequence spaces and their matrix maps, Publ. Inst. Math. (Beograd) 42 (1987), 57-61.

[10] E. Malkowsky, Absolute and ordinary Köthe-Toeplitz duals of some sets of sequences and matrix transformations, Publ. Inst. Math., (Beograd)(NS), 46(60) (1989), 97-103.

[11] B. Choudhary, S. K. Mishra, A note on certain sequence spaces, J. Anal., 1 (1993), 139-148.

[12] S. K. Mishra, Matrix maps involving certain sequence spaces, Indian J. Pure Appl. Math., 24(2) (1993), $125-132$.

[13] M. Mursaleen, A. K. Gaur, A. H. Saifi,Some new sequence spaces and their duals and matrix transformations, Bull. Calcutta Math. Soc., 88(3) (1996), 207-212.

[14] C. Gnanaseelan, P. D. Srivastava, The $\alpha-, \beta$ - and $\gamma$-duals of some generalised difference sequence spaces, Indian J. Math., 38(2) (1996), 111-120.

[15] E. Malkowsky, A note on the Köthe-Toeplitz duals of generalized sets of bounded and convergent difference sequences, J. Anal., 4 (1996), $981-91$.

[16] A. K. Gaur, M. Mursaleen, Difference sequence spaces, Int. J. Math. Math. Sci., 21(4) (1998), 701-706.

[17] E. Malkowsky, M. Mursaleen, Qamaruddin, Generalized sets of difference sequences, their duals and matrix transformations, In: Sequence Spaces and App. Narosa, New Delhi, 1999, pp. 68-83.

[18] Ç. Asma, R. Çolak, On the Köthe-Toeplitz duals of some generalized sets of difference sequences, Demonstratio Math., 33 (2000), $797-803$.

[19] E. Malkowsky, M. Mursaleen, Some matrix transformations between the difference sequence spaces $\Delta c_{0}(p)$, $\Delta c(p)$ and $\Delta \ell_{\infty}(p)$, Filomat, 15 (2001),

[20] M. Kirişçi, F. Başar, Some new sequence spaces derived by the domain of generalized difference matrix, Comput. Math. Appl., 60(5) (2010), 1299-1309.

[21] A. Sönmez, A. Some new sequence spaces derived by the domain of the triple band matrix, Comput. Math. Appl., 62(2) (2011), 641-650.

[22] M. Candan, Domain of the double sequential band matrix in the classical sequence spaces, J. Inequal. Appl., 281(1) (2012), 1-15.

[23] M. Candan, Almost convergence and double sequential band matrix, Acta Math. Sci. Ser. B, 34(2) (2014) 354-366.

[24] M. Candan, Domain of the double sequential band matrix in the spaces of convergent and null sequences, Adv. Differ. Equ., 163(1) (2014), 1-18.

[25] M. Stieglitz, H. Tietz, Matrix transformationen von folgenräumen eine ergebnisübersicht, Math. Z., 154 (1977), 1-16.

[26] K.-G. Grosse-Erdmann, On $\ell^{1}$-invariant sequence spaces, J. Math. Anal. Appl., 262 (2001), 112-132.

[27] B. Altay, F. Başar, Certain topological properties and duals of the matrix domain of a triangle matrix in a sequence space, J. Math. Anal. Appl., 336(1) (2007), 632-645.

[28] F. Başar, B. Altay, On the space of sequences of p-bounded variation and related matrix mappings, Ukrainian Math. J., 55(1) (2003), $136-147$.

[29] F. Yaşar, K. Kayaduman, A different study on the spaces of generalized Fibonacci difference bs and cs spaces sequence, Symmetry, 10 (2018), 274, doi:10.3390/sym10070274.

[30] K. Kayaduman, F. Yaşar, On domain of Nörlund matrix Mathematics, 6 (2018), 268, doi:10.3390/math6110268.

[31] F. Yaşar, K. Kayaduman, On the domain of the Fibonacci difference matrix, Mathematics, 7 (2019), 204, doi:10.3390/math7020204.

[32] K. Kayaduman, F. Yaşar, A. Çetin, On some inequalities and $\sigma^{B(r, s)}$-conservative matrices, J. Inequal. Speci. Func., 9(2) (2018), 82-91.

[33] M. Candan, K. Kayaduman, Almost convergent sequence space derived by generalized Fibonacci matrix and Fibonacci core, British J. Math. \& Computer Sci., 7 (2015), 150-167.

[34] M. İlkhan, E. E. Kara, A new Banach space defined by Euler totient matrix operator, Oper. Matrices, 13(2) (2019), 527-544. 
[35] E. E. Kara, Some topological and geometrical properties of new Banach sequence spaces, J. Inequal. Appl., 38 (2013), https://doi.org/10.1186/1029242X-2013-38

[36] E. E. Kara, M. İlkhan, Some properties of generalized Fibonacci sequence spaces, Linear Multilinear Algebra, 64(11) (2016), 2208-2223, 1145626.

[37] M. Kirişçi, The application domain of infinite matrices with algorithms, Uni. J. Math. Appl., 1(1) (2018), 1-9.

[38] F. Başar, M. Kirişçi, Almost convergence and generalized difference matrix, Comput. Math. Appl., 61(3) (2011), 602-611. 